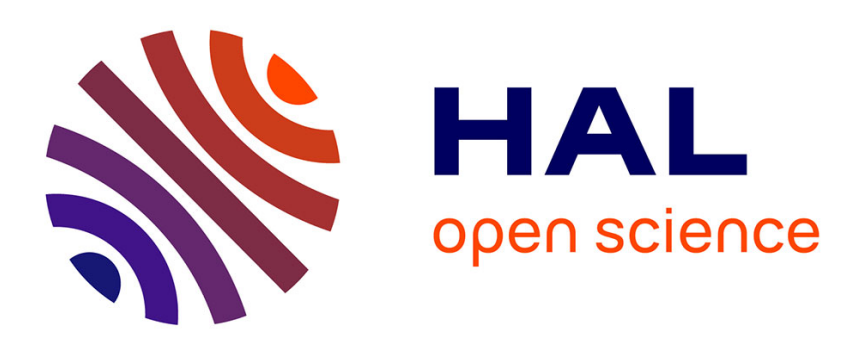

\title{
Effect of solvent on silicon nanoparticle formation and size: a mechanistic study
}

Sanaa Semlali, Benoit Cormary, Maria de Marco, Jérôme Majimel, Alix

Sournia-Saquet, Yannick Coppel, Mathieu Gonidec, Patrick Rosa, Glenna L. Drisko

\section{To cite this version:}

Sanaa Semlali, Benoit Cormary, Maria de Marco, Jérôme Majimel, Alix Sournia-Saquet, et al.. Effect of solvent on silicon nanoparticle formation and size: a mechanistic study. Nanoscale, 2019, 11 (11), pp.4696-4700. 10.1039/C9NR00619B . hal-02071674

\section{HAL Id: hal-02071674 https://hal.science/hal-02071674}

Submitted on 18 Mar 2019

HAL is a multi-disciplinary open access archive for the deposit and dissemination of scientific research documents, whether they are published or not. The documents may come from teaching and research institutions in France or abroad, or from public or private research centers.
L'archive ouverte pluridisciplinaire HAL, est destinée au dépôt et à la diffusion de documents scientifiques de niveau recherche, publiés ou non, émanant des établissements d'enseignement et de recherche français ou étrangers, des laboratoires publics ou privés. 


\section{Effect of solvent on silicon nanoparticle formation and size: a mechanistic study}

Sanaa Semlali, ${ }^{a}$ Benoit Cormary, ${ }^{a *}$ Maria L. De Marco, ${ }^{a}$ Jérôme Majimel, ${ }^{a}$ Alix Saquet, ${ }^{b}$ Yannick Coppel, ${ }^{b}$ Mathieu Gonidec, ${ }^{\text {a }}$ Patrick Rosa, ${ }^{a}$ Glenna L. Drisko ${ }^{a *}$

Silicon has emerged as the most desirable material for optics, however chemists struggle to control its morphology and dimensions for larger diametres. Here the average diametre of silicon nanoparticles is varied between 3 and $15 \mathrm{~nm}$ by changing the reaction solvent. Electrochemistry and NMR elucidate the role of solvent on the synthetic mechanism. Surprisingly the solvent does not stabilize the nanoparticles and there is no trend associated with chain length or open-chain versus cyclical solvent molecules. The solvents' main role is to stabilize the by-products, which prelongs the reaction lifetime.

Silicon has exceptional optical properties spanning from photoluminescence to Mie resonance, which has generated frenetic activity among physicists in recent years. ${ }^{1}$ Silicon is an abundant and nontoxic element, thus commercial applications are expected from optically active biomedical probes to telecommunications. ${ }^{1-3}$ One particularly exciting application is the prospect of forward-light scattering, which can be used for beam shaping. In pursuit of optical metamaterials, large particle diameters, that is $\geq 75 \mathrm{~nm}$, are required. ${ }^{1}$ It is especially difficult to grow nanoparticles larger than $10 \mathrm{~nm}$ using solution chemistry. The size restriction can be attributed to the sluggish particle growth kinetics compared to nucleation rate. It is wellknown that a strong reducing agent favours fast nucleation, consuming most of the precursor. ${ }^{4}$ As small nanoparticles are typically obtained by strong reductants, ${ }^{5}$ a gentle reductant is required to promote particle growth over nucleation. Magnesium ( $\mathrm{Mg}$ ) is a gentler reducing agent than the others commonly used, i.e. naphthalenides, alkalides, metal hydrides and Zintl salts. This synthetic protocol is similar to one published by S. Kauzlarich and coworkers, where $\mathrm{SiCl}_{4}$ was reduced by the Zyntl salt $\mathrm{Mg}_{2} \mathrm{Si}^{6}{ }^{6}$ This metathesis reaction producing particles in glyme with a diameter between $2-5 \mathrm{~nm},{ }^{6}$ where the $\mathrm{Mg}$ reduction reported here generates particles around $10 \mathrm{~nm}$. However, $\mathrm{Mg}$ does not reduce $\mathrm{SiCl}_{4}$ in all solvents, ${ }^{7}$ begging two questions: What is the reduction mechanism? And what is the solvent's role in this reaction?

Scheme 1. Formation mechanism of Si NPs (blue spheres). The Mg (grey sphere) is not drawn to scale, being millimetric in size, where the silicon is nanometric. Clusters of $\mathrm{Si}$ are represented by a blue $\mathrm{Y}$, the $\mathrm{MgCl}_{2}$ as white crystals.

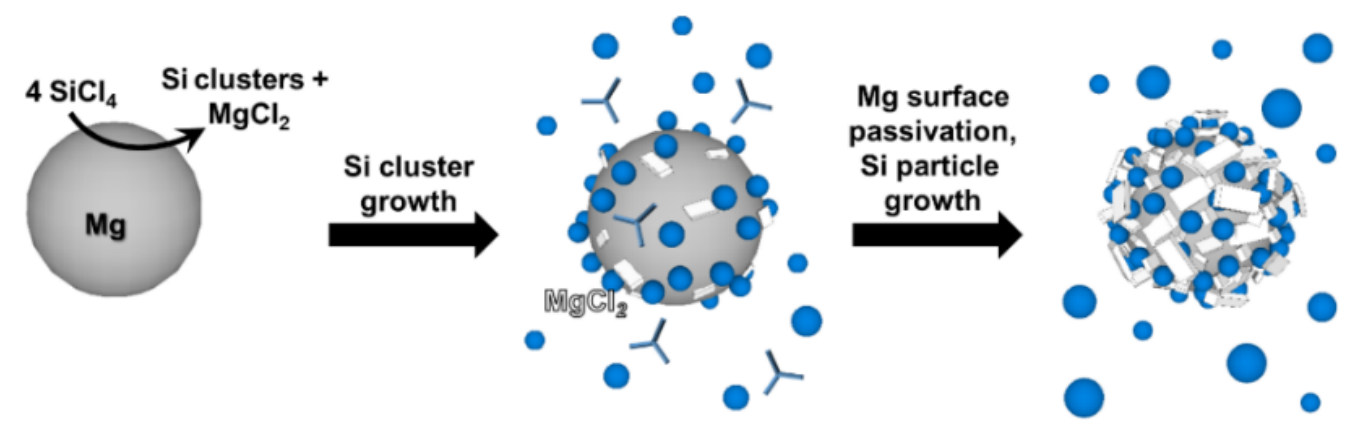



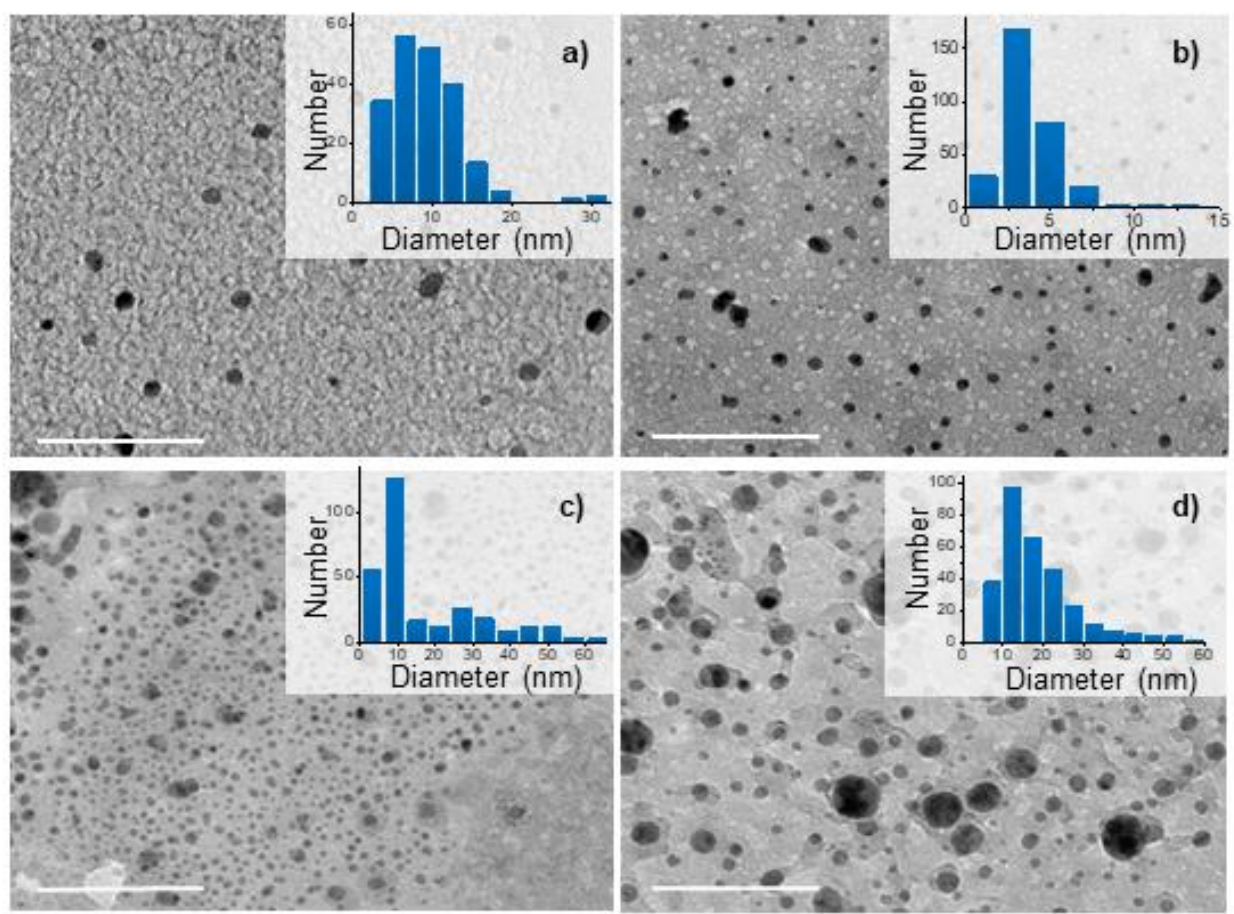

Fig. 1 Bright field TEM and particle size distribution of Si NPs synthesized in (a) THF, (b) diglyme, (c) glyme, and (d) tetraglyme. Scale bars represent $100 \mathrm{~nm}$.

No one has yet studied the solvent effect on silicon nanoparticle (Si NP) size, even though the solvent changes the reactivity of reducing agents and precursors, while either stabilizing the nanoparticles or promoting their aggregation. For example, in the preparation of CdS nanoparticles, the particle size was ten times smaller in pure alcohol than in an alcohol/water mixture. ${ }^{8}$ Here we tested six solvents, in the form of glycol ethers and heterocyclic ethers. We have chosen to use $\mathrm{Mg}$ as a reductant, due to its relatively mild reactivity and because it does not pollute the silicon structure, as hydrogen can. ${ }^{9}$ Using $\mathrm{Mg}$ and $\mathrm{SiCl}_{4}$, we were able to vary the mean nanoparticle size between 3-15 $\mathrm{nm}$ simply by changing the solvent. This reaction is relatively easy, as it occurs at room temperature and ambient pressure, using commercially available products. It is also easy to handle and poses less security risks than most other reducing agents. We have investigated the reaction mechanism using NMR and by determining the reduction potentials in the respective solvents. $\mathrm{SiCl}_{4}$ was reduced by $\mathrm{Mg}$ grit at room temperature and ambient pressure in the presence of THF, glyme, diglyme and tetraglyme. The reaction had little induction time, with a visible colour change occurring within a few minutes of mixing the reagents. Reduction did not occur in diethyl ether and dioxane. A previous report claims that the reaction does not occur in toluene or anisole. ${ }^{7}$
By producing Si NPs under different conditions and studying the synthesis using solution-state NMR and electrochemistry, we propose that these nanoparticles form via the mechanism shown in Scheme 1. $\mathrm{SiCl}_{4}$ reduction is observed through a colour change, from colourless to yellow, to red, to brown, as an evolution in nanoparticle size. $\mathrm{The}_{\mathrm{SiCl}}$ reduction occurs at the Mg surface. We can eliminate a mechanism based on the formation of silanylmagnesium complexes, the analogue of the carbon-based Grignard reagent, a mechanism that was proposed previously. ${ }^{7}$ Silanylmagnesium compounds cannot be produced from chloride-based silicon precursors at room temperature, and if they did form, they would cleave the glycol ether solvents. ${ }^{10}$ At the onset of the reaction, the $\mathrm{Mg}$ grit is covered by a thin oxide layer. Upon $\mathrm{SiCl}_{4}$ addition, the surface of the $\mathrm{Mg}$ is partially scrubbed, generating a very small amount of $\mathrm{SiO}_{2}$ and a reactive magnesium surface. The $\mathrm{SiCl}_{4}$ then grows into clusters of several silicon atoms, generating $\mathrm{MgCl}_{2}$ as a byproduct. The vast majority of the silicon clusters and the $\mathrm{MgCl}_{2}$ detach from the surface of the $\mathrm{Mg}$ grit. However, as time progresses, the surface eventually becomes encumbered with a skin of salt and Si NPs. Elemental analysis of pieces of Mg grit at the end of the reaction in different solvents was conducted using a scanning electron microscope (SEM) (Fig. S1, S2 ESI). The surface was covered with two types of zones: One rich in silicon and one rich in both magnesium and chloride. This skin 


\section{COMMUNICATION}
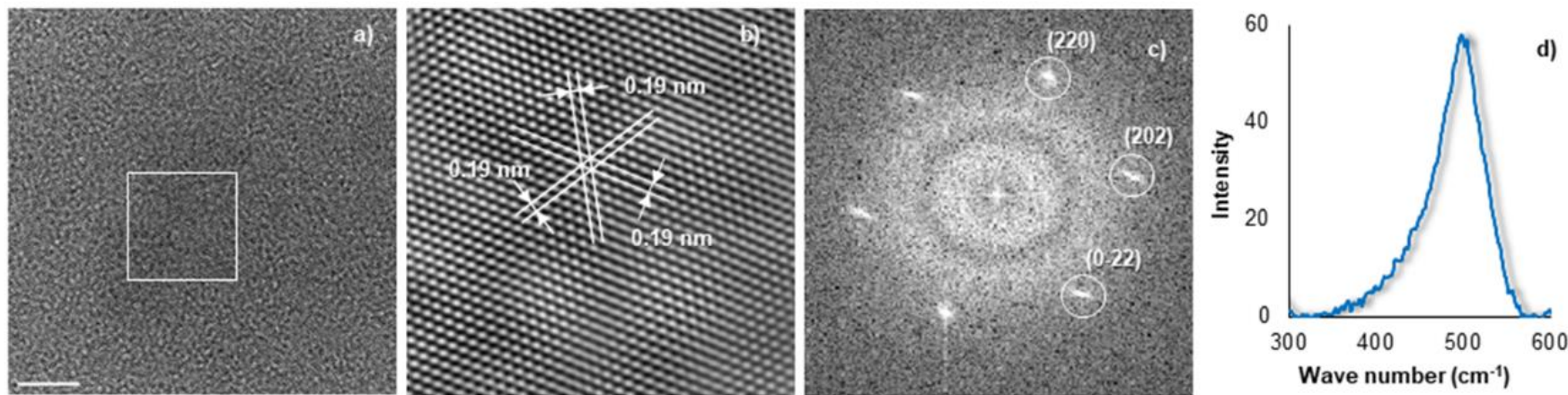

Fig. 2 (a) HRTEM (scale bar = $5 \mathrm{~nm}$ ), (b) filtered HRTEM image, (c) SAED, and (d) Raman spectroscopy of a nanoparticle produced in glyme.

passivates the Mg surface and prevents further reaction, even if $\mathrm{SiCl}_{4}$ still exists in the solution, terminating nanoparticle growth. The solvent does not intervene directly in the reaction. We believe that the solvent's main effect on the final size of the Si NPs is due to its capacity to solvate $\mathrm{MgCl}_{2}$ and $\mathrm{Si}$ species, and its physical properties (e.g. viscosity, reduction potential of the $\mathrm{SiCl}_{4}$, polarity and density). NMR and electrochemistry studies provide evidence supporting this mechanism.

By simply changing the solvent from one glycol ether to another, the Si NP size distribution is significantly altered. Fig. 1 shows representative TEM images and histograms of the size distribution of Si NPs synthesized in four different glycol ethers. The results are not immediately logical, the trend in size is not linearly connected to the number of ether units, and oddly THF produces particles where its linear analogue, diethyl ether, does not. The smallest Si NPs were obtained in diglyme, having an average diameter of $6 \pm 2 \mathrm{~nm}$. Si NPs synthesized in THF have a size centred around $8 \pm 8 \mathrm{~nm}$. The synthesis in glyme offers a large range of Si NP sizes: from 5 to $60 \mathrm{~nm}$; however half of the NPs have a diameter between 5 and $10 \mathrm{~nm}$. In tetraglyme, a size maximum distribution occurs at $15 \mathrm{~nm}$, with a substantial quantity of particles being larger than $20 \mathrm{~nm}$. Perhaps the substantially larger size observed in tetraglyme is due to its viscosity, slowing NP diffusion away from the reactive surface. Pure, crystalline Si NPs are produced by this room temperature method, as confirmed with high resolution transmission electron microscopy (HRTEM) and Raman spectroscopy (Fig. 2). The Si NPs contain diffraction planes, visible using HRTEM (Fig. 2a). The d-spacing for the planes in this orientation were 0.19 $\mathrm{nm}$, which correspond to the $\{220\} \mathrm{hkl}$ plane for the diamond cubic structure. The selected area electron diffraction (SAED) pattern indexes to the [-111] zone axis of cubic silicon, the expected crystallographic structure. Si nanoparticles with a chlorinated surface become charged by observation under the electron beam and thus leave the TEM grid after $\sim 6$ of exposure time. This short window of observation did not allow us to do EDX spectra of the silicon nanoparticles. However, we performed electron diffraction on particles of all sizes and consistently found that the particles were silicon. The Raman spectrum of the Si NPs possesses a peak at $510 \mathrm{~cm}^{-1}$ that corresponds to crystalline silicon. ${ }^{11}$ No other peaks were observed, indicating the purity of the sample. Thus, crystalline silicon can be successfully produced using this room temperature reaction.

Analytic electrochemistry was used to probe the observed trend in Si NP size as a function of solvent (Fig. 3). The reductive potential of the Mg fluctuated rather dramatically according to the solvent used. The starting potential $\left(\mathrm{E}_{\mathrm{t}=0}\right)$ indicates the role of the solvent on the electrode. The starting potentials measured in glycol ethers are similar to the potential observed for a $\mathrm{Mg}$ electrode in $\mathrm{DMF}$ with $\mathrm{NBu}_{4} \mathrm{Cl}$ as the electrolyte salt. ${ }^{12}$ Upon $\mathrm{SiCl}_{4}$ addition, an initial oxidation occurs. The oxidation is likely due to the reaction of $\mathrm{SiCl}_{4}$ with the passivating oxide layer present at the surface of the $\mathrm{Mg}$ electrode. This initial oxidation is observed in all of the solvents studied. In the case of dioxane, once the oxidation is complete, the silicon precursor no longer reacts with $\mathrm{Mg}$. The starting potential in dioxane is $1.4 \mathrm{~V} / \mathrm{SCE}$.

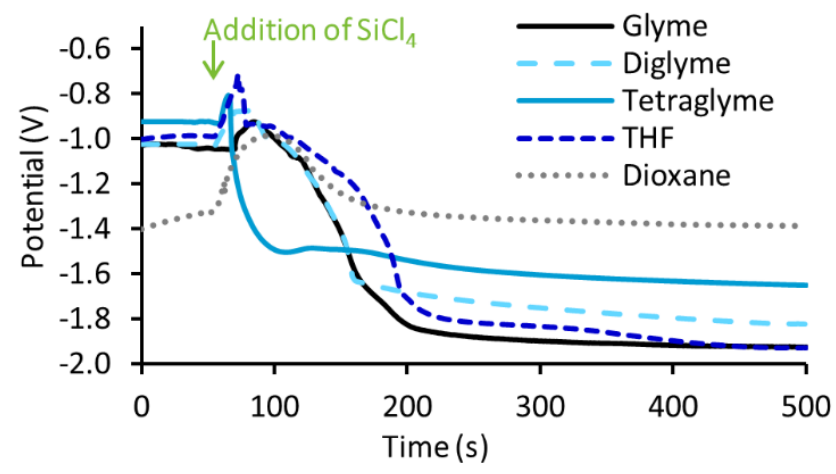

Fig. 3 Chronopotentiometry measurement using a Mg electrode of the equilibrium potential in various solvents before and after adding $\mathrm{SiCl}_{4}$.

Although dioxane shows the lowest potential in the presence of $\mathrm{Mg}$ and absence of $\mathrm{SiCl}_{4}$, after addition of $\mathrm{SiCl}_{4}$, dioxane shows the highest potential of the set and does not form nanoparticles. In the other solvents studied, the reaction was 
spontaneous. After $\mathrm{SiCl}_{4}$ injection, there are different reduction kinetics. Within $200 \mathrm{~s}$, the potential stabilizes at a lower value. The final potential value ranged from -1.6 to $-2.0 \mathrm{~V} / \mathrm{SCE}$, and is probably the standard potential of the $\mathrm{MgCl}_{2} / \mathrm{Mg}$ couple.

Upon cycling the $\mathrm{Mg}$ electrode in the presence of $\mathrm{SiCl}_{4}$, in all solvents except dioxane (Fig. S3, ESI) the surface became highly resistive and eventually passivated. Thus, no further reaction between $\mathrm{Mg}$ and $\mathrm{SiCl}_{4}$ was possible. The passivation results from the side product of the reduction, which is probably the magnesium chloride salt. ${ }^{13}$ In dioxane, the Mg surface remained unchanged (Fig. S3b, ESI). When platinum was used as the working electrode in place of $\mathrm{Mg}$, the $\mathrm{SiCl}_{4}$ was not reduced (Fig. S4, ESI). Thus, platinum does not intervene in the reaction in the same way as $\mathrm{Mg}$ does. The electrochemistry confirms what was observed synthetically, no nanoparticles are formed when dioxane is employed. Moreover, the reduction in the other solvents is spontaneous on the surface of the $\mathrm{Mg}$. While differences in the redox potential of $\mathrm{Mg} / \mathrm{MgCl}_{2}$ appear to induce noticeable differences in the final sizes of Si NPs in each solvent, the relationship between solvent and reductant is not univocal: diglyme yielded smaller sizes than THF and glyme while having a higher potential. It is likely that other factors, such as viscosity, come into play.

NMR experiments help shed light upon the interaction between the solvent and the nanoparticles and show which species are present in solution. In situ growth NMR studies were performed without agitation and as a result, the reduction occurred much slower than the reactions taking place in a Schlenk flask.

When monitoring the reaction over time using ${ }^{29} \mathrm{Si} N \mathrm{NMR}$ spectroscopy, at the onset only $\mathrm{SiCl}_{4}$ is present $(\delta-18 \mathrm{ppm}, \mathrm{Fig}$ $\mathrm{S} 5 \mathrm{ESI})$. After $4 \mathrm{~h}$, peaks appeared at 0 and $30 \mathrm{ppm}$. The peak at $0 \mathrm{ppm}$ later disappears, indicating that it is a relatively stable intermediate species, which is then consumed. At longer times, a peak is still observed at $30 \mathrm{ppm}$, but with a strongly increased width. F. Meyer-Wegner, et al. have published the chemical shifts for $\mathrm{Si}$ in $\mathrm{SinCl}_{2 \mathrm{n}+2}$ species of $\mathrm{n}=1-5 .{ }^{14}$ These species can only be detected if they are free in solution, species bound to the $\mathrm{Mg}$ grit cannot be observed. The peaks that we observe do not correspond to those reported by Meyer-Wegner and coworkers, thus we believe that they correspond to clusters larger than 5 silicon atoms. Alternatively, however, these downfield shifts may correspond to $\mathrm{SiCl}_{2}$ silylene adducts: examples of ternary amine adducts are reported in the literature in cleavage reactions of chlorosilanes in the presence of reductants, so solvent adducts in our likewise reducing conditions could be a possibility. There is little evolution after $6 \mathrm{~h}$, indicating that the $\mathrm{Mg}$ surface is no longer active. The $\mathrm{SiCl}_{4}$ peak at $-18 \mathrm{ppm}$ is consistently present. Another peak appears at $-128 \mathrm{ppm}$ after 4 $\mathrm{h}$ of reaction, which then shifts to -140 ppm by $6 \mathrm{~h}$. After $46 \mathrm{~h}$, two new peaks appear at -39 and $-46 \mathrm{ppm}$, which we cannot explain at this time. The peaks are only present when THF is used as a solvent, thus it is perhaps due to a degraded THF molecule attacking a silicon chloride species, as silicates can appear in this region of the spectrum. After a second addition of $\mathrm{Mg}$, these three peaks disappear, which is probably because the THF becomes so crosslinked that it is no longer sufficiently mobile to be detected by liquid NMR. The solution had gellified by the end of the reaction. The mechanism is further clarified using ${ }^{35} \mathrm{Cl}$ NMR.

The consumption of the $\mathrm{SiCl}_{4}$ precursor is monitored using ${ }^{35} \mathrm{Cl}$ NMR (Fig. 4). It is possible to observe the ${ }^{35} \mathrm{Cl}$ signal due to the tetrahedral geometry of the chloride, which averages to zero the quadrupolar coupling. Chlorides occurring in other geometries are not observable. At the beginning of the experiment, $\mathrm{SiCl}_{4}$ is added to 4 equivalents of $\mathrm{Mg}$ grit in deuterated THF. The intensity of the $\mathrm{Cl}$ peak initially decreases at a linear rate, and then stabilizes. Twenty\% of the initial concentration of $\mathrm{SiCl}_{4}$ is consumed after $5.5 \mathrm{~h}$. At this point, a fresh quantity of $\mathrm{Mg}$ (4 equivalents) is added. The $\mathrm{SiCl}_{4}$ reacts with the fresh $\mathrm{Mg}$ and stabilizes by $8 \mathrm{~h}$. By this time, $62 \%$ of the $\mathrm{SiCl}_{4}$ has been consumed. For comparison, in the case of a reaction with just one single addition of 10 equivalents of $\mathrm{Mg}$ grit, the $\mathrm{SiCl}_{4}$ concentration decreases by only $35 \%$. The second addition of $\mathrm{Mg}$ yields a faster consumption of the silicon precursor. The same behaviour is observed in the case of 2 successive additions of 2 equivalents of $\mathrm{Mg}$ (Fig. S6, ESI). These results illustrate that a successive addition of reductant is more efficient than a single addition. The NMR experiments confirm the electrochemistry results: the reaction occurs rapidly until the $\mathrm{Mg}$ surface becomes passivated and the reaction stops.

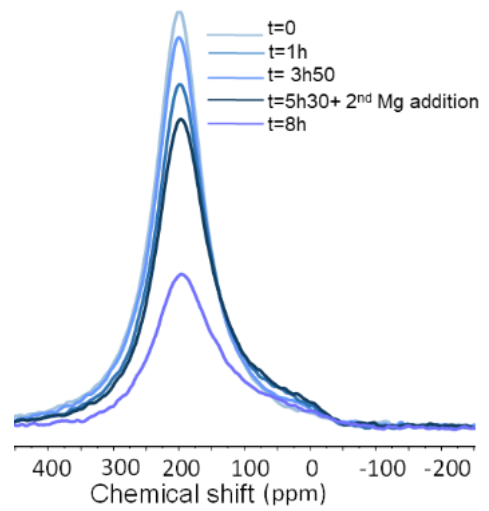

Fig. $4{ }^{35} \mathrm{Cl} \mathrm{NMR}$ of $\mathrm{SiCl}_{4}$ and $\mathrm{Mg}$ grit in THF-D8 at different time intervals and with 4 equivalents of $\mathrm{Mg}$.

${ }^{1} \mathrm{H}$ NMR studies show no visible species, with the exception of THF (data not shown). THF shows a minor chemical shift over time, probably due to its polymerization. The NMR studies show no interaction between the solvent and the growing Si NPs, indicating that the solvent molecules are not in fast exchange between a surface-bound and solvated state, and thus it is unlikely that the solvent is behaving as a ligand as has been observed for other semiconductor nanoparticles. ${ }^{15}$ Solvent does not seem to stabilize the formed Si NPs. Thus, the changing particle size as a function of solvent must be mainly due to its effect on the reduction potential of $\mathrm{SiCl}_{4}$ and its capacity to solvate $\mathrm{MgCl}_{2}$ and Si species.

Physicists challenge chemists to develop silicon particles, but the difficulty of working with silicon leads to slow progress. In pursuit of a bottom-up chemistry synthesis of Si NPs large enough to support a Mie resonance, it is important to examine 
each reaction parameter separately: the silicon precursor, the reducing agent, the solvent and the reaction conditions. Here, solvent was the subject of study, where tetraglyme proved to produce nanoparticles much larger on average than in other glycol ethers. The $\mathrm{Mg}$ reduction of $\mathrm{SiCl}_{4}$ was studied because $\mathrm{Mg}$ is a gentle reducing agent, which should favour growth over nucleation. In all cases, the Si NPs obtained were cubic crystalline silicon. The $\mathrm{Mg}$ donates its electrons without polluting the silicon structure. Electrochemistry and NMR experiments indicate that the reaction occurs quickly and spontaneously on the $\mathrm{Mg}$ surface. $\mathrm{Mg}$ reduction did provide larger nanoparticle sizes, however polydispersity in nanoparticle size was large. Thus, Mg may not be the right reductant for solution-based routes to optically resonant silicon nanoparticles. Although silicon-based optical metamaterials still cannot be achieved using solution chemistry, this work shows that solvent needs to be considered in order to maximize nanoparticle size.

\section{Conflicts of interest}

There are no conflicts to declare.

\section{Notes and references}

SS, BC, MLDM and GLD were supported by the LabEx AMADEus (ANR-10-LABX-42). They and MG acknowledge the framework of IdEx Bordeaux (ANR-10-IDEX-03-02), i.e. the Investissements $d$ 'Avenir program of the French government managed by the Agence Nationale de la Recherche. PLACAMAT (UMS 3626CNRS-Bordeaux University) provided technical support with imaging. FEGSEM and EDX instrumentation was facilitated by the Institut des Matériaux de Paris Centre (IMPC FR2482) and was funded by Sorbonne Université, CNRS and by the C'Nano projects of the Région Ile-de-France. We thank David Montero for performing the FEGSEM and EDX analysis.

1 M. L. De Marco, S. Semlali, B. A. Korgel, P. Barois, G. L. Drisko and C. Aymonier, Angew. Chem. Int. Ed., 2018, 57, 4478.

2 R. Dezert, P. Richetti, A. Baron, J. Phys.: Conf. Series 2018, 1092, 012022

3 I. Staude, J. Schilling, Nat. Photonics 2017, 11, 274.

4 N. T. K. Thanh, N. Maclean and S. Mahiddine, Chem. Rev., 2014, 114, 7610.

5 A. Shiohara, S. Prabakar, A. Faramus, C.-Y. Hsu, P.-S. Lai, P. T. Northcote and R. D. Tilley, Nanoscale, 2011, 3, 3364.

6 C.-S. Yang, R. A. Bley, S. M. Kauzlarich, H. W. H. Lee, G. R. Delgado, J. Am. Chem. Soc. 1999, 121, 5191.

7 Y. Chen, G. Yang, R. Zhao and W. Xue, Mater. Sci. Forum, 2014, 809-810, 180.

8 P. Thakur and S. S. Joshi, J. Exp. Nanosci., 2012, 7, 547.

9 L. Shi, J. Harris, R. Fenollosa, I. Rodriguez, X. Lu, B. A. Korgel and F. Meseguer, Nat. Commun., 2013, 4, 1904.

10 R. Goddard, C. Krüger, N. A. Ramadan and A. Ritter, Angew. Chem. Int. Ed., 1995, 34, 1030.

11 C. Meier, S. Lüttjohann, V. G. Kravets, H. Nienhaus, A. Lorke, H. Wiggers, Physica E 2006, 32, 155.

12 J. Serp, PhD, Université Paul Sabatier- Toulouse III, 1999.
13 Z. Lu, A. Schechter, M. Moshkovich and D. Aurbach, J. Electroanal. Chem., 1999, 466, 203.

14 F. Meyer-Wegner, A. Nadj, M. Bolte, N. Auner, M. Wagner, M. C. Holthausen and H.-W. Lerner, Chem. Eur. J., 2011, 17, 4715.

15 M. Branca, K. Corp, D. Ciuculescu-Pradines, Y. Coppel, P. Lecante and C. Amiens, New J. Chem., 2017, 41, 5960.

\section{TOC graphic}

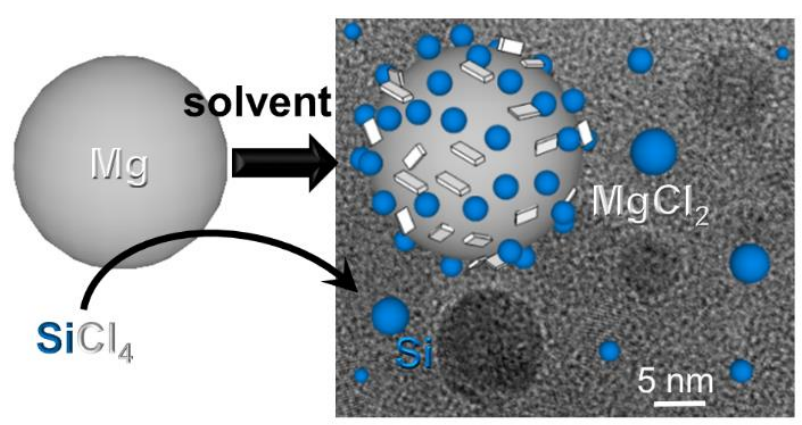

Silicon nanoparticle size is adjusted by simply changing the reaction solvent. The role of solvent on nanoparticle formation is investigated. 